\title{
Sensitivity analysis of viscoelastic structures
}

\author{
A.M.G. de Lima ${ }^{\mathrm{a}}$, M.H. Stoppa ${ }^{\mathrm{b}}$, D.A. Rade ${ }^{\mathrm{a}, *}$ and V. Steffen. Jr. ${ }^{\mathrm{a}}$ \\ ${ }^{\text {a }}$ Federal University of Uberlândia, School of Mechanical Engineering, Brazil \\ ${ }^{\mathrm{b}}$ Federal University of Goiás, Department of Mathematics Campus Santa Mônica, P.O. Box 593, CEP 38400-902, \\ Uberlândia, $M G$, Brazil
}

\begin{abstract}
In the context of control of sound and vibration of mechanical systems, the use of viscoelastic materials has been regarded as a convenient strategy in many types of industrial applications. Numerical models based on finite element discretization have been frequently used in the analysis and design of complex structural systems incorporating viscoelastic materials. Such models must account for the typical dependence of the viscoelastic characteristics on operational and environmental parameters, such as frequency and temperature. In many applications, including optimal design and model updating, sensitivity analysis based on numerical models is a very usefull tool. In this paper, the formulation of first-order sensitivity analysis of complex frequency response functions is developed for plates treated with passive constraining damping layers, considering geometrical characteristics, such as the thicknesses of the multi-layer components, as design variables. Also, the sensitivity of the frequency response functions with respect to temperature is introduced. As an example, response derivatives are calculated for a three-layer sandwich plate and the results obtained are compared with first-order finite-difference approximations.
\end{abstract}

Keywords: Viscoelasticity, finite elements, plates, sensitivity analysis, damping

\section{Introduction}

Passive damping approaches to the problem of vibration attenuation constitute an important subject in modern Engineering. The use of viscoelastic materials to reduce noise and vibration levels in various types of engineering systems such as robots, automobiles, airplanes, buildings and space structures has been intensively investigated lately. Much of the knowledge available to date is compiled in the books by Nashif et al. [1] and Mead [2] and in some review papers such as those by Rao [3] and Samali and Kwok [4].

In the last two decades, a great deal of effort has been devoted to the development of mathematical models for characterizing the mechanical behavior of viscoelastic materials, accounting for its typical dependence on operational and environmental factors, such as excitation frequency, static preloads and temperature. Besides the well-known complex modulus approach, which constitutes a simple and straightforward means of representing the dynamic behavior of viscoelastic materials in frequency domain [1], some other models have been suggested and have been shown to be particularly suitable to be used in combination with finite element discretization, which makes them very attractive for the modeling of complex engineering systems. Among those models, it should be mentioned the so-called Fractional Derivative Model (FDM) [5-7], the Golla-Hughes-McTavish Model (GHM) [8,9] and the Anelastic Displacement Fields Model (ADF) suggested by Lesieutre and co-workers [10-12]. All these models can represent the viscoelastic behavior both in time and frequency domain. The modeling by finite elements of industrial structures containing viscoelastic surface treatments and mounts has been recently addressed in references [13-15]. Moreover, viscoelastic models have been used in the study of other strategies of vibration control such as active constraing layer damping [16,17] and vibration neutralizers [18].

It has been recognized that temperature exerts the most noticeable environmental effect on the mechanical behavior of viscoleastic materials. For themorheologically-simple viscoelastic materials, the influence of temperature can be

\footnotetext{
*Corresponding author. Tel.: +55 34323941 48; Fax: +55 34323942 06; E-mail: domingos@ufu.br.
} 
accounted for based on the principle of the correspondence frequency-temperature, which leads to the concept of reduced frequency [19].

In the context of analysis and design of structural systems, an important topic to be addressed is the so-called sensitivity analysis, which enables to evaluate the degree of influence of variations of physical and/or geometrical parameters on the mechanical behavior. Sensitivity analysis constitutes an important step in various types of problems such as model updating, analysis of modified structures, optimal design, system identification, control and stochastic reliability assessment [20-22].

Sensitivity analysis is generally based on the evaluation of the derivatives (most frequently limited to the first order) of the system response with respect to a set of parameters of interest. It can be associated to different kinds of mechanical responses: static displacements, eigenvalues and eigenvectors, frequency response functions and time responses [20]. According to Murthy and Haftka [23], the optimal design structural systems has a narrow connection with sensitivity analysis, since a significant part of typical optimization algorithms generally perform a large number of evaluations of the system response for different values of the design variables. Derivatives can be used to approximate the response of modified systems, thus reducing the cost of re-analysis, especially for high-order systems. The same applies for finite element stochastic modeling of structures, which requires a large amount of computations to characterize uncertainty propagation [24].

Several approaches have been developed for performing sensitivity analysis of dynamic responses, as reported in references [20,25-27]. However, applications to the case of structural systems containing viscoelastic components are not numerous, which motivates the study reported herein.

In this remainder, sensitivity analysis is applied to plates treated with surface constraining damping layer treatments, considering, as dynamic responses, the complex frequency response functions (FRFs). With this aim, after a brief review of the fundamental concepts of viscoelasticity, a finite element model of a three-layer sandwich plate is developed in combination with the complex modulus approach to account for the frequency-dependent behavior of the viscoelastic layer. The influence of temperature is introduced by using the concept of reduced frequency. Then, the formulation of the sensitivities of the FRFs with respect to geometrical parameters and temperature is developed. For illustration, this formulation is used to compute the sensitivities of FRFs of a rectangular plate partially treated with a constrained viscoelastic layer. For validation, the results obtained are compared to first-order finite-difference approximations.

\section{The complex modulus approach}

According to the linear theory of viscoelasticity [19], the one-dimensional stress-strain relation can be expressed in Laplace domain as follows:

$$
\sigma(s)=G(s) \varepsilon(s)
$$

where:

$$
G(s)=G_{r}+H(s)
$$

In the equation above, $G_{r}$ is the static modulus, representing the elastic behavior and $H(s)$ is the relaxation function, associated to the dissipation effects. When evaluated along the imaginary axis of the s-plane $(s=i \omega)$, Eq. (2) leads to the complex modulus, which can be expressed as follows:

$$
\begin{aligned}
& G(\omega)=G^{\prime}(\omega)+i G^{\prime \prime}(\omega) \\
& \eta(\omega)=G^{\prime \prime}(\omega) / G^{\prime}(\omega)
\end{aligned}
$$

where $G^{\prime}(\omega), G^{\prime \prime}(\omega)$ and $\eta(\omega)$ are defined, respectively, as storage modulus, loss modulus and loss factor of the viscoelastic materials. Any pair formed by these three parameters completely characterizes the dynamic characteristics of viscoelastic materials in the frequency domain. 


\section{Influence of temperature on the viscoelastic behavior}

Temperature is usually considered to be the most important environmental factor which exerts influence upon the properties of viscoelastic materials. Thus, it becomes important to account for temperature variations in the modeling of structural systems containing viscoelastic elements. According to Nashif et al. [1], this can be done by making use of the so-called Frequency-Temperature Superposition Principle - FTSP, which establishes a relation between the effects of the excitation frequency and temperature on the proprieties of the thermorheologically simple viscoelastic materials. This implies that the viscoelastic characteristics at different temperatures can be related to each other by changes (or shifts) in the actual values of the excitation frequency. This leads to the concepts of shift factor and reduced frequency. Symbolically, the FTSP can be expressed under the following forms:

$$
\begin{aligned}
& G(\omega, T)=G\left(\omega_{r}, T_{z}\right)=G\left(\alpha_{T} \omega, T_{z}\right) \\
& \eta\left(\omega_{r}, T\right)=\eta\left(\alpha_{T} \omega, T_{z}\right)
\end{aligned}
$$

where $\omega_{r}=\alpha_{T}(T) \omega$ is the reduced frequency, $\omega$ is the actual frequency, $\alpha_{T}(T)$ is the shift function that must be identified from experimental tests for each type of viscoelastic material, and $T_{z}$ is a reference value of temperature.

Figure 1 illustrates the FTSP, showing that having the modulus and loss factor of an arbitrary viscoelastic material for different temperature values, $T_{-1}, T_{z}, T_{1}$, if horizontal shifts along the frequency axis are applied to each of these curves, all of them can be combined into a single one. The horizontal shift is given by $\alpha_{T}$ and depends on the temperature.
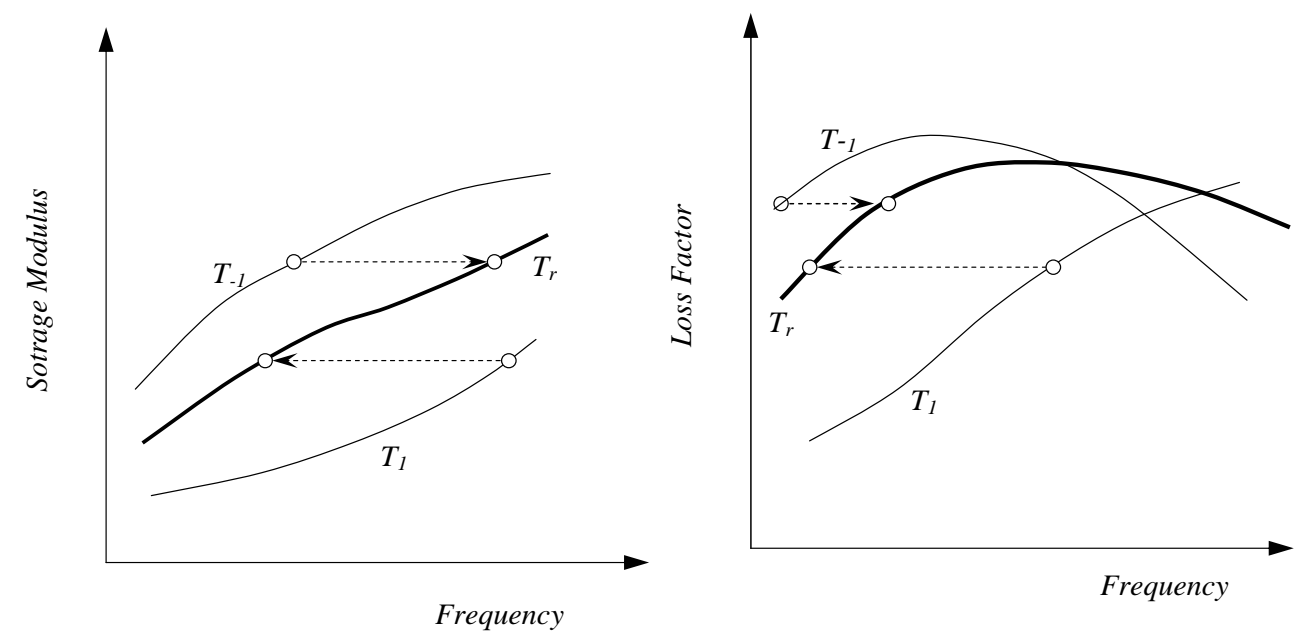

Fig. 1. Illustration of the Frequency-Temperature Superposition Principle.

\section{Viscoelastic behavior incorporated into finite element models}

The finite element equations of motion of a viscoelastic structure containing $N$ degrees of freedom can be expressed as follows:

$$
M \ddot{q}(t)+C \dot{q}(t)+K(\omega, T) q(t)=f(t)
$$

where $M, C, K(\omega, T) \in R^{N \times N}$ are the mass (symmetric, positive-definite), viscous damping (symmetric, nonnegative-definite), and stiffness (symmetric, nonnegative-definite) matrices. $q(t), f(t) \in R^{N}$ are the vectors of displacements and external loads, respectively.

It is assumed that the model contains both elastic and viscoelastic elements, so that the stiffness matrix can be decomposed as follows: 


$$
K(\omega, T)=K_{e}+K_{v}(\omega, T)
$$

where $K_{e}$ is the stiffness matrix corresponding to the purely elastic substructure and $K_{v}(\omega, T)$ is the stiffness matrix associated with the viscoelastic substructure. The inclusion of the frequency-dependent behavior of the viscoelastic material can be made by using the so-called elastic-viscoelastic correspondence principle [8], according to which, for a given temperature, $K_{v}(\omega, T)$ can be first generated for specific types of finite elements (rods, beam, plates, etc.) assuming that the longitudinal modulus and/or the shear modulus (according to the stress-state) are frequency-independent. Then, after the finite element matrix is constructed, the frequency-dependence of such moduli is introduced according to a particular viscoelastic constitutive model adopted. By assuming the widely accepted hypothesis of a constant (frequency-independent) Poisson ratio for the viscoelastic material, $E(\omega, T)$ becomes proportional to $G(\omega, T)$ through the relation $G(\omega, T)=E(\omega, T) / 2(1+\nu)$. Then, one of the two moduli can be factored-out of the stiffness matrix of the viscoelastic sub-structure and one writes:

$$
K_{v}(\omega, T)=G(\omega, T) \bar{K}_{v}
$$

Equations (7), (8) and (9) can be combined to give:

$$
M \ddot{q}(t)+C \dot{q}(t)+\left(K_{e}+G(\omega, T) \bar{K}_{v}\right) q(t)=f(t)
$$

The difficulty in dealing with Eq. (10) for predicting the vibration response in the time domain and performing eigenvalue analysis comes from the fact that the stiffness matrix depends on frequency. Some procedures for dealing with this problem have been suggested, based on the adoption of particular representations for the frequencydependent behavior of the viscoelastic materials. Such an approach is used in the Fractional Derivative, GollaHughes-McTavish and Anelastic Displacement Field models (see references in Section 1), which enable to transform Eq. (10) in state-space form, with constant (frequency-independent) state matrices, at the expense of a typically high increase in the order of the system matrices.

The interest herein is focused on frequency domain responses. In this case, Eq. (10) can be directly used for calculating the steady-state harmonic responses in the frequency domain, by assuming:

$$
\begin{aligned}
& f(t)=F e^{i \omega t} \\
& q(t)=Q(\omega) e^{i \omega t}
\end{aligned}
$$

Upon introduction of Eqs (10) and (11) into Eq. (10), the following relation is obtained between the amplitudes of the excitation forces and the amplitudes of the harmonic responses:

$$
Q(\omega)=H(\omega, T) F
$$

where the receptance or frequency response function (FRF) matrix is expressed as:

$$
H(\omega, T)=\left[-\omega^{2} M+i \omega C+K_{e}+G(\omega, T) \bar{K}_{v}\right]^{-1}
$$

Based on Eq. (13), the interest is to evaluate the sensitivities of FRFs with respect to physical and/or geometrical parameters featuring in the finite element matrices and also with respect to temperature. Such approach will be applied to plates treated with passive constraining layer damping, whose finite element discretization is summarized in the next section.

\section{A three-layer sandwich plate finite element}

In this section the formulation of a three-layer sandwich plate finite element is summarized, based on the original development made by Kathua and Cheung [28]. Figure 2 depicts a rectangular element formed by an elastic baseplate, a viscoelastic core and an elastic constraining layer, whose dimensions in directions $x$ and $y$ are denoted by $a$ and $b$, respectively. Superscripts (1), (2) and (3) will be used to identify the quantities pertaining the base-plate, the viscoelastic core and the constraining layer, respectively.

Space discretization is made by considering 4 nodes and 7 degrees-of-freedom per node, indicated in Fig. 2, representing the nodal in-plane displacements of the base-plate middle plane in directions $x$ and $y$ (denoted by $u_{1} \mathrm{e}$ 


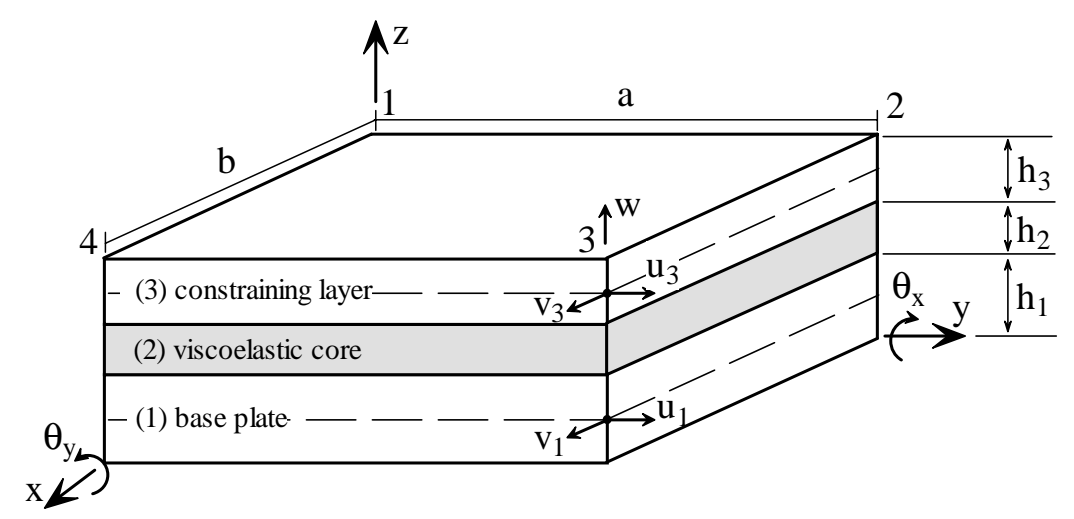

Fig. 2. Three-layer sandwich plate finite element.

$v_{1}$ ), the nodal in-plane displacements of the constraining layer middle plane in directions $x$ and $y$ (denoted by $u_{3}$ and $v_{3}$ ), the nodal transverse displacement, $w$, and the nodal cross-section rotations about axes $x$ and $y$, denoted by $\theta_{x}$ and $\theta_{y}$, respectively.

The vectors of nodal and element degrees-of-freedom are then expressed as follows:

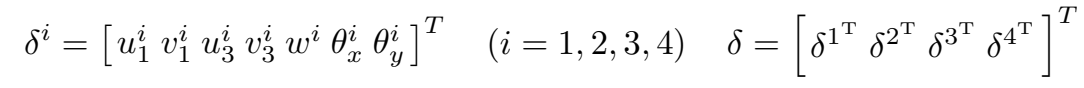

In the development of the theory, the following assumptions are adopted:

- normal stresses and strains in direction $z$ are neglected for all the three layers.

- the elastic layers (base-plate and constraining layer) are modeled according to Kirchhoff's theory, which neglects the effects associated to transverse (thickness-wise) shear;

- for the viscoelastic core transverse shear in considered;

- the transverse displacement $w$ is the same for all the three layers and cross-section rotations $\theta_{x}$ and $\theta_{y}$ are assumed to be the same for the elastic layers (1) and (3).

By imposing kinematic relations that enforce the continuity of displacements along the interfaces between the layers, the following expressions are found for the longitudinal displacements for points on the middle plane of the viscoelastic core in directions $x$ and $y$ in terms of the previously defined nodal coordinates:

$$
u_{2}=\frac{1}{2}\left[u_{1}+u_{3}+\frac{h_{1}-h_{3}}{2} \frac{\partial w}{\partial x}\right], \quad v_{2}=\frac{1}{2}\left[v_{1}+v_{3}+\frac{h_{1}-h_{3}}{2} \frac{\partial w}{\partial y}\right]
$$

The longitudinal and transverse displacements are interpolated within the element as follows:

$$
\begin{aligned}
& u_{1}=u_{1}(x, y)=a_{1}+a_{2} x+a_{3} y+a_{4} x y \quad v_{1}=v_{1}(x, y)=a_{5}+a_{6} x+a_{7} y+a_{8} x y \\
& u_{3}=u_{3}(x, y)=a_{9}+a_{10} x+a_{11} y+a_{12} x y \quad v_{3}=v_{3}(x, y)=a_{13}+a_{14} x+a_{15} y+a_{18} x y \\
& w=w(x, y)=b_{1}+b_{2} x+b_{3} y+b_{4} x^{2}+b_{5} x y+b_{6} y^{2}+b_{7} x^{3}+b_{8} x^{2} y+\cdots \\
& \cdots+b_{9} x y^{2}+b_{10} y^{3}+b_{11} x^{3} y+b_{12} x y^{3}
\end{aligned}
$$

Based on the hypotheses of the stress-states assumed for each layer, and isotropic mechanical properties, the following stress-strain relations apply:

- For the base-plate:

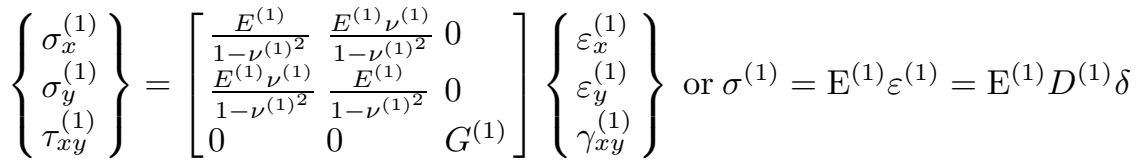


- For the viscoelastic core:

$$
\begin{aligned}
& \left\{\begin{array}{l}
\sigma_{x}^{(2)} \\
\sigma_{y}^{(2)} \\
\tau_{x y}^{(2)} \\
\tau_{x z}^{(2)} \\
\tau_{y z}^{(2)}
\end{array}\right\}=\left[\begin{array}{lllll}
\frac{E^{(2)}(\omega, T)}{1-\nu^{(2)}} & \frac{E^{(2)}(\omega, T) \nu^{(2)}}{\left.1-\nu^{(2)}\right)^{2}} & 0 & 0 & 0 \\
\frac{E^{(2)}(\omega, T) \nu^{(2)}}{1-\nu^{(2)^{2}}} & \frac{E^{(2)}(\omega, T)}{1-\nu^{(2)^{2}}} & 0 & 0 & 0 \\
0 & 0 & G^{(2)}(\omega, T) & 0 & 0 \\
0 & 0 & 0 & G^{(2)}(\omega, T) & 0 \\
0 & 0 & 0 & 0 & G^{(2)}(\omega, T)
\end{array}\right]\left\{\begin{array}{l}
\varepsilon_{x}^{(2)} \\
\varepsilon_{y}^{(2)} \\
\gamma_{x y}^{(2)} \\
\gamma_{x z}^{(2)} \\
\gamma_{y z}^{(2)^{2}}
\end{array}\right\} \text { or } \\
& \sigma^{(2)}=\mathrm{E}^{(2)}(\omega, \mathrm{T}) \varepsilon^{(2)}=\mathrm{E}^{(2)}(\omega, \mathrm{T}) D^{(2)} \delta
\end{aligned}
$$

- For the constraining layer:

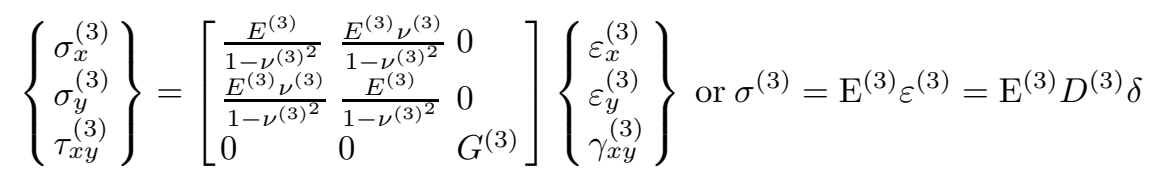

where matrices $D^{(\kappa)}(k=1,2,3)$ are formed by derivatives of the shape functions according to the differential operators appearing in the strain-displacement relations for each layer. Details are given by Kathua and Cheung [28] and Stoppa [29]. Based on the stress-strain relations above, neglecting viscous damping, the strain and kinetic energies of the composite plate element are formulated. Lagrange equations are used, considering the nodal displacements and rotations as generalized coordinates, to obtain the element stiffness and mass matrices. Such procedure has been fully developed using symbolic computation by Stoppa [29].

According to Eqs (8) and (9), after assembling, the stiffness matrix can be expressed as follows:

For the elastic sub-structure: $K_{e}=K^{(1)}+K^{(3)}$

For the viscoelastic substructure: $K_{v}(\omega, T)=K^{(2)}(\omega, T)=G(\omega, T) \bar{K}^{(2)}$

where matrices $K^{(1)}, K^{(3)}$ and $K^{(2)}(\omega, T)$ are, respectively, the contributions to the stiffness matrices of the base-plate, constraining layer and viscoelastic core.

The finite element model of the three-layer sandwich plate has been validated by comparison with experimental results in reference [29], which provides a comparison between numerical and experimental FRFs of a rectangular plate partially treated with passive constraining layer damping.

\section{Sensitivity analysis of structural responses}

The global finite element matrices, $M, C$ and $K(\omega, T)$ appearing in Eq. (7) establish the dependence of the response of the system with respect to a set of design parameters, which include physical and geometrical characteristics and temperature. Such functional dependence can be expressed in a general form as follows:

$$
r=r(M(p), C(p), K(p))
$$

where $r$ and $p$ designate vectors of structural responses and design parameters, respectively.

The sensitivity of the structural responses with respect to a given parameter $p_{i}$, evaluated for a given set of values of the design parameters $p^{0}$ is defined as the following partial derivative:

$$
\left.\frac{\partial r}{\partial p_{i}}\right|_{p^{0}}=\lim _{\Delta p_{i} \rightarrow 0}\left[\frac{r\left(M\left(p_{i}^{0}+\Delta p_{i}\right), C\left(p_{i}^{0}+\Delta p_{i}\right), K\left(p_{i}^{0}+\Delta p_{i}\right)\right)}{\Delta p_{i}}-\frac{r\left(M\left(p_{i}^{0}\right), C\left(p_{i}^{0}\right), K\left(p_{i}^{0}\right)\right)}{\Delta p_{i}}\right]
$$

where $\Delta p_{i}$ is an arbitrary variation applied to the current value of parameter $p_{i}^{0}$, while all other parameters remain unchanged.

The sensitivity with respect to $p_{i}$ can be estimated by finite differences by computing successively the responses corresponding to $p_{i}=p_{i}^{0}$ and $p_{i}=p_{i}^{0}+\Delta p_{i}$ and then computing: 


$$
\left.\frac{\partial r}{\partial p_{i}}\right|_{p_{i}^{0}} \approx\left[\frac{r\left(M\left(p_{i}^{0}+\Delta p_{i}\right), C\left(p_{i}^{0}+\Delta p_{i}\right), K\left(p_{i}^{0}+\Delta p_{i}\right)\right)}{\Delta p_{i}}-\frac{r\left(M\left(p_{i}^{0}\right), C\left(p_{i}^{0}\right), K\left(p_{i}^{0}\right)\right)}{\Delta p_{i}}\right]
$$

Such approach is in general inefficient from the computation point of view. Moreover, the results depend upon the choice of the value of the parameter increment $\Delta p_{i}$. Another strategy consists in computing the analytical derivatives of the structural responses with respect to the parameters of interest. This approach is considered in the following section.

\subsection{Sensitivity of frequency response functions with respect to structural parameters}

Consider the FRF matrix of a viscoelastically damped system as given by Eq. (14). Sensitivity with respect to a given structural parameter $p_{i}$ can be computed by deriving the relation $H(\omega, T, p) H^{-1}(\omega, T, p)=I$, which leads to the following expression:

$$
\left.\frac{\partial H(\omega, T, p)}{\partial p_{i}}\right|_{\left(\omega, T^{0}, p^{0}\right)}=-H\left(\omega, T^{0}, p^{0}\right)\left(-\omega^{2} \frac{\partial M\left(p^{0}\right)}{\partial p_{i}}+i \omega \frac{\partial C\left(p^{0}\right)}{\partial p_{i}}+\frac{\partial K\left(\omega, T^{0}, p^{0}\right)}{\partial p_{i}}\right) H\left(\omega, T^{0}, p^{0}\right)
$$

Regarding the equation above, it should be noted that when parameter $p_{i}$ appears explicitly in matrices $M$ and/or $C$, and/or $K$ (which is frequently the case), the computation of the derivatives of these matrices with respect to such parameter is straightforward, generally resulting in sparse matrices.

\subsection{Sensitivity of the frequency response functions with respect to temperature}

The computation of the derivatives of FRFs with respect to temperature requires that such parameter appear explicity in the stiffness matrix of the viscoelastic substructure. With this aim, a procedure based on the use of the frequency-temperature equivalence principle and the concept of reduced frequency (addressed in Section 3) can be used.

By combining Eqs (5) and (13) with Eq. (24), one writes:

$$
\begin{aligned}
& H(\omega, T)=\left[-\omega^{2} M+i \omega C+K_{e}+G\left(\omega_{r}, T_{z}\right) \bar{K}_{v}\right]^{-1} \\
& \left.\frac{\partial H(\omega, T, p)}{\partial T}\right|_{\left(\omega, T^{0}, p^{0}\right)}=-H\left(\omega_{r}, T_{z}, p^{0}\right)\left[\frac{\partial G\left(\omega_{r}, T_{z}\right)}{\partial T} \bar{K}_{v}\left(p^{0}\right)\right] H\left(\omega_{r}, T_{z}, p^{0}\right)
\end{aligned}
$$

The derivative of the complex modulus with respect to temperature can be computed as follows:

$$
\frac{\partial G\left(\omega_{r}, T_{z}\right)}{\partial T}=\frac{\partial G}{\partial \omega_{r}} \frac{\partial \omega_{r}}{\partial T}=\frac{\partial G}{\partial \omega_{r}} \frac{\partial \alpha_{T}}{\partial T} \omega
$$

Functions $G\left(\omega_{r}\right)$ and $\alpha_{T}(T)$ can be obtained from experimental tests for specific viscoelastic materials. Some of these functions are proposed Nashif et al. [1]. Also, as the result of a comprehensive experimental work, Drake and Soovere suggest analytical expressions for the complex modulus and shift factor for various commercially available viscoelastic materials, as functions of reduced frequency and temperature [30]. The following equations represent, respectively, the complex modulus and shift factor as functions of temperature and reduced frequency for the $3 \mathrm{M}$ ISD $112^{\mathrm{TM}}$ viscoelastic material (which is considered in the numerical applications that follow), as provided by those authors:

$$
\begin{aligned}
& G\left(\omega_{r}\right)=B_{1}+B_{2} /\left(1+B_{5}\left(i \omega_{r} / B_{3}\right)^{-B_{6}}+\left(i \omega_{r} / B_{3}\right)^{-B_{4}}\right) \\
& \log \left(\alpha_{T}\right)=a\left(\frac{1}{T}-\frac{1}{T_{z}}\right)+2.303\left(\frac{2 a}{T_{z}}-b\right) \log \left(\frac{T}{T_{z}}\right)+\left(\frac{b}{T_{z}}-\frac{a}{T_{z}^{2}}-S_{A Z}\right)\left(T-T_{z}\right)
\end{aligned}
$$


Table 1

Physical and geometrical properties of FE model of the three-layer plate

\begin{tabular}{lcccc}
\hline Layer & Thickness $(\mathrm{m})$ & $\begin{array}{c}\text { Yong modulus } \\
\left(\mathrm{N} / \mathrm{m}^{2}\right)\end{array}$ & $\begin{array}{c}\text { Poisson } \\
\text { ratio }\end{array}$ & $\begin{array}{c}\text { Density } \\
\left(\mathrm{kg} / \mathrm{m}^{3}\right)\end{array}$ \\
\hline Constraining & $0.5 \times 10^{-3}$ & $70.3 \times 10^{9}$ & 0.345 & 2750 \\
Viscoelastic & $20.0 \times 10^{-5}$ & According to Eqs $(28)$ and $(29)$ & 0.500 & 1099.5 \\
Base-plate & $3.0 \times 10^{-3}$ & $70.3 \times 10^{9}$ & 0.345 & 2750 \\
\hline
\end{tabular}

where:

$$
\begin{aligned}
& B_{1}=0.4307 \mathrm{MPa} ; B_{2}=1200 \mathrm{MPa} ; B_{3}=0.1543 \mathrm{MPa} ; B_{4}=0.6847 ; B_{5}=3.2410 ; B_{6}=0.1800 ; \\
& T_{z}=290 \mathrm{~K} ; T_{L}=210 \mathrm{~K} ; T_{H}=360 \mathrm{~K} ; S_{A Z}=0.05956 \mathrm{~K}^{-1} ; S_{A L}=0.1474 \mathrm{~K}^{-1} ; \\
& S_{A H}=0.009725 \mathrm{~K}^{-1} ; \\
& a=\left(\left(D_{B} C_{C}-C_{B} D_{C}\right) / D_{E}\right) ; b=\left(D_{C} C_{A}-C_{C} D_{A}\right) / D_{E} ; C_{A}=\left(1 / T_{L}-1 / T_{Z}\right)^{2} \\
& C_{B}=\left(1 / T_{L}-1 / T_{Z}\right) ; C_{c}=S_{A L}-S_{A Z}
\end{aligned}
$$

Figure 3 depicts the standardized curves representing the variations of the storage modulus, loss modulus and loss factor as functions of the reduced frequency, as obtained from Eq. (28), and a plot of the shift factor as a function of the temperature, as given by Eq. (29).
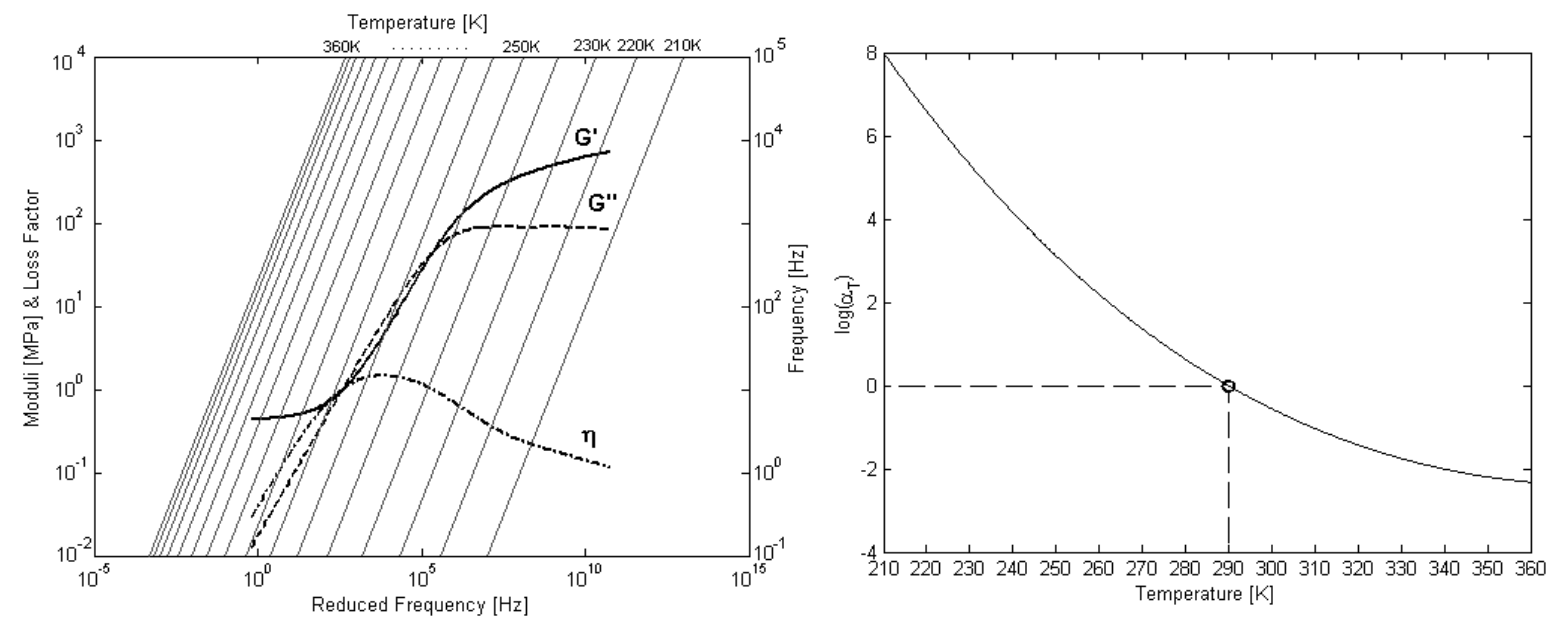

Fig. 3. Master and shift factor curves for the $3 \mathrm{M}^{\mathrm{TM}}$ ISD112 viscoelastic material.

\section{Numerical applications to plates treated with constrained viscoelastic layer}

To illustrate the procedure of computation of the sensitivity of FRFs, numerical tests were performed using the FE model of a freely suspended plate made of aluminum, fully treated with a layer of 3M ISD112 ${ }^{\mathrm{TM}}$ viscoelastic material constrained between the base plate and an outer aluminum sheet as shown in Fig. 4. The FE model, whose physical and geometrical characteristics are given in Table 1, has a total number of 80 finite elements, 99 nodes and 693 degrees-of-freedom. The computations consisted in obtaining the sensitivities of the driving point FRF corresponding to the point $I$ indicated on Fig. 4, which are denoted by $H_{I I}(\omega, T, p)$. 


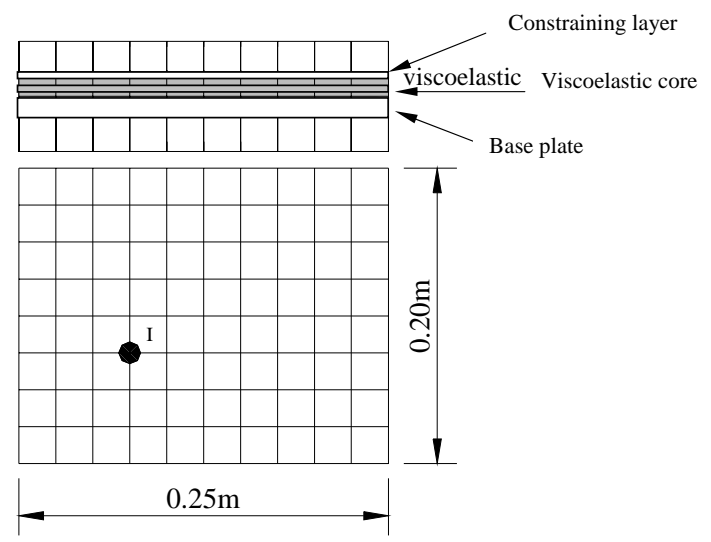

Fig. 4. Illustration of the FE model of a free plate with full surface treatment.
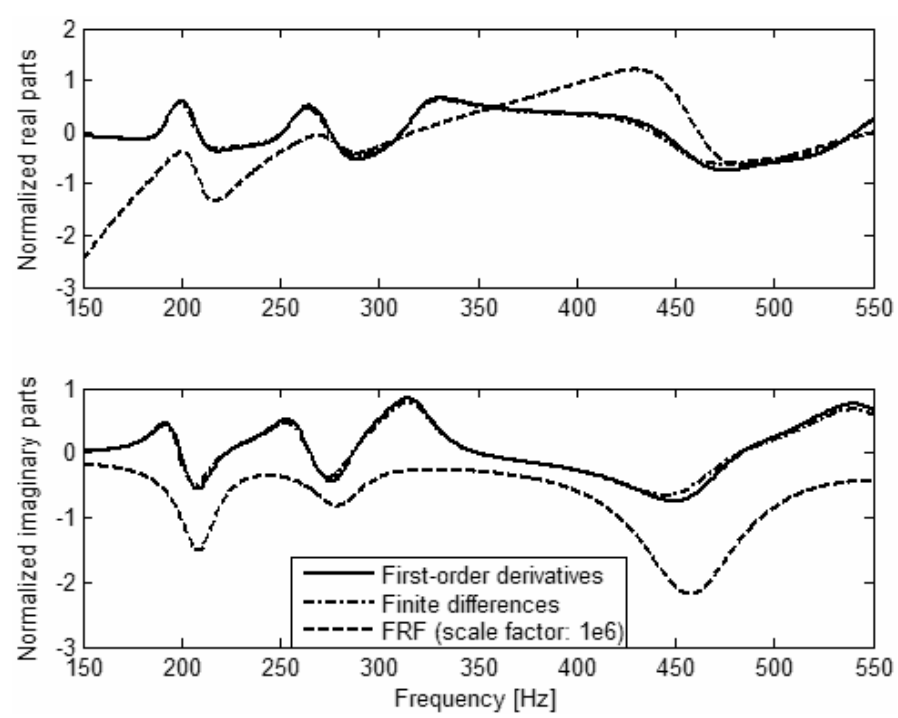

Fig. 5. Sensitivities of the FRF $H_{I I}(\omega, T, p)$ with respect to the thickness of the viscoelastic layer.

\subsection{Sensitivities of the FRF with respect to structural parameters}

In this example, the thicknesses of the constraining and viscoelastic layers were considered as the design variables in the computation of the sensitivities of the FRF $H_{I I}(\omega, T, p)$. The real and imaginary parts of the complex sensitivity functions obtained by using the first-order derivatives according to Eq. (24) are shown in Figs 5 and 6 , where they are compared to the approximate sensitivity functions calculated by finite differences (according to Eq. (23) having been adopted a variation from $20 \%$ of the nominal values of the parameters given in Table 1 . Also, in the same figure, the real and imaginary parts of the FRF $H_{I I}(\omega, T, p)$, multiplied by convenient scale factors, are shown. It is to be noted that the sensitivity functions, denoted by $S_{F R F}^{N}(\omega, T, p)$, have been normalized according to the following scheme:

$$
S_{F R F}^{N}(\omega, T, p)=\left.\frac{\partial H_{I I}(\omega, T, p)}{\partial p}\right|_{\omega, T, p_{0}} \frac{p_{0}}{\left|H I I\left(\omega, T, p_{0}\right)\right|}
$$

Figures 5 and 6 enable to compare the computed first-order derivatives with the results obtained by first-order finite differences. In addition, based on the amplitudes and sign of the sensitivity functions one can evaluate the degree of influence of the design variables upon the amplitudes of the FRF in the frequency band considered. 

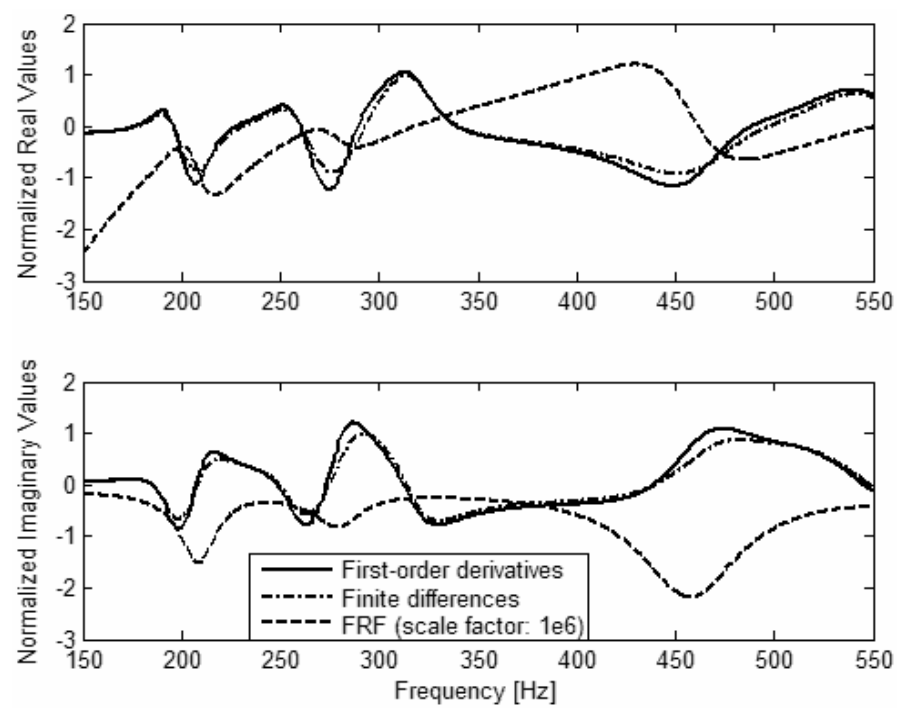

Fig. 6. Sensitivities of the FRF $H_{I I}(\omega, T, p)$ with respect to the thickness of the constraining layer.

\subsection{Sensitivity of FRF with respect to temperature}

For the computation of the derivative of the FRF $H_{I I}(\omega, T, p)$ with respect to temperature, according to Eq. (26), the derivatives indicated in Eq. (27) were computed by deriving Eqs (28) and (29) with respect to $\omega_{r}$ and $T$, which lead to the following expressions:

$$
\begin{aligned}
\frac{\partial G}{\partial \omega_{r}}= & B_{2} B_{3}^{B_{6}}\left(B_{5} B_{6} e^{-\frac{i B_{6} \pi}{2}}\left(1 / \omega_{r}\right)^{B_{6}+1}+B_{4} e^{-\frac{i B_{4} \pi}{2}}\left(1 / \omega_{r}\right)^{B_{4}+1}\right) / \\
& \left(1+B_{5} e^{-\frac{i B_{6} \pi}{2}}\left(B_{3} / \omega_{r}\right)^{B_{6}}+e^{-\frac{i B_{4} \pi}{2}}\left(B_{3} / \omega_{r}\right)^{B_{4}}\right)^{2} \\
\frac{\partial \alpha_{T}}{\partial T}= & \alpha_{T}\left(-\frac{a}{T^{2}}+2.303\left(\frac{2 a}{T_{z}}-b\right) \frac{\log e}{T}+\frac{b}{T_{z}}-\frac{a}{T_{z}^{2}}-S_{A Z}\right) \ln 10
\end{aligned}
$$

Figures 7 and 8 show the normalized real and imaginary parts of the sensitivity functions of the FRF $H_{I I}(\omega, T, p)$ with respect to temperature, for two different values of the nominal temperature, as compared to their counterparts calculated by finite differences, using variations of $2 \%$ of the nominal temperature values. Here again, the real and imaginary parts of the FRF $H_{I I}(\omega, T, p)$, multiplied by a convenient scaling factor are also presented. In these figures, it can be seen that the first-order derivatives compare fairly well with finite differences and enable to evaluate the degree of influence of temperature variations within the frequency band of interest.

As a complementary demonstration of the utility of first-order derivatives in the analysis of modified structures, the FRF $H_{I I}(\omega, T, p)$ of the three-layer sandwich plate was computed in two different forms: in the first, given a set of nominal values of the design variables (viscoelastic layer thickness, constraining layer thickness, both given in Table 1, and temperature $T=298 \mathrm{~K}$ ), variations were voluntarily applied to these values ( $15 \%$ for both layer thicknesses and $1.5 \%$ for the temperature values). Then, the exact FRFs of the perturbed system were computed. In the second form, the FRFs of the perturbed system were estimated from the FRFs of the nominal system by using the first order derivatives computed according to the formulation presented in the previous sections, as follows:

$$
H_{I I}\left(\omega, T, p_{0}+\Delta p\right) \cong H_{I I}\left(\omega, T, p_{0}\right)+\left.\frac{\partial H_{I I}(\omega, T, p)}{\partial p}\right|_{p=p_{0}}
$$



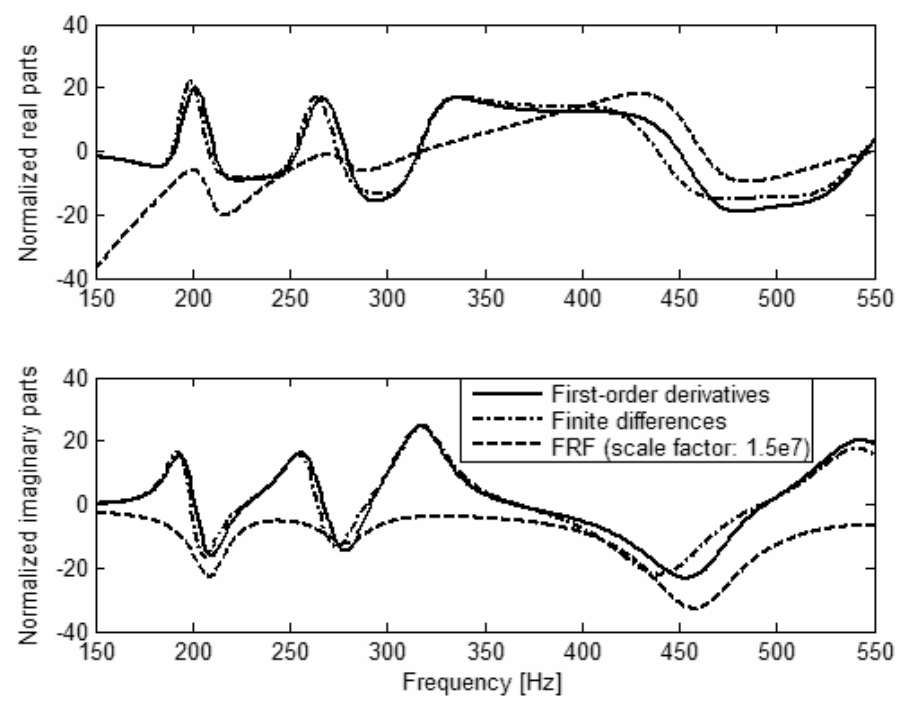

Fig. 7. Sensitivities of the FRF $H_{I I}(\omega, T, p)$ with respect to temperature for a nominal temperature of $298 \mathrm{~K}$.
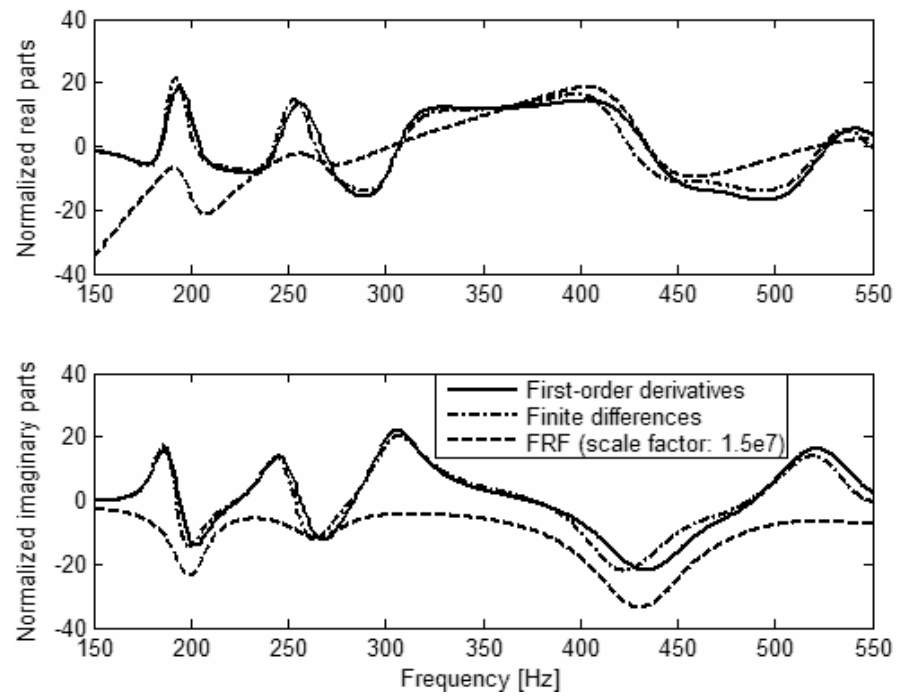

Fig. 8. Sensitivities of the FRF $H_{I I}(\omega, T, p)$ with respect to temperature for a nominal temperature of $308 \mathrm{~K}$.

$$
H_{I I}(\omega, T+\Delta T, p) \cong H_{I I}\left(\omega, T_{0}, p\right)+\left.\frac{\partial H_{I I}(\omega, T, p)}{\partial T}\right|_{T=T_{0}}
$$

As can be seen in Figs 9 to 11, the exact FRFs are fairly accurately approximated by the first-order series expansions. The agreement is less satisfactory for the temperature variations, leading to conclude that approximations based on first-order derivatives might not be accurate enough to predict variations of the dynamic behavior of the viscoelastic materials associated to larger variations of the temperature. In this case, the results could possibly be improved by using second-order Taylor series expansions which require the knowledge of both first-order and second-order derivatives. 

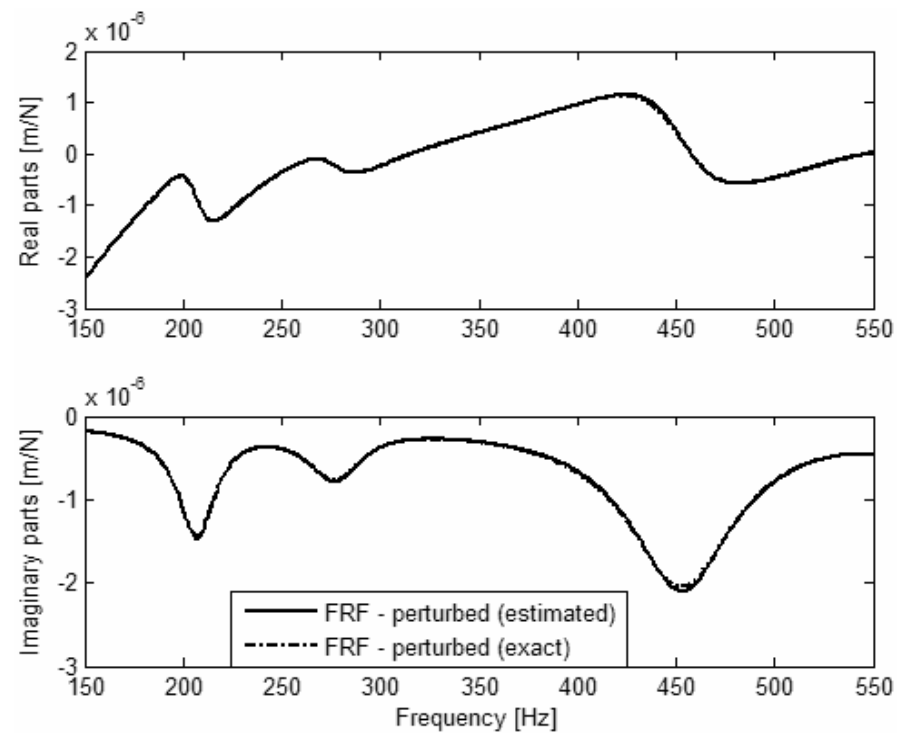

Fig. 9. Exact and first-order estimation of the FRF $H_{I I}(\omega, T, p)$ of the perturbed system by variations of the viscoelastic layer thickness.
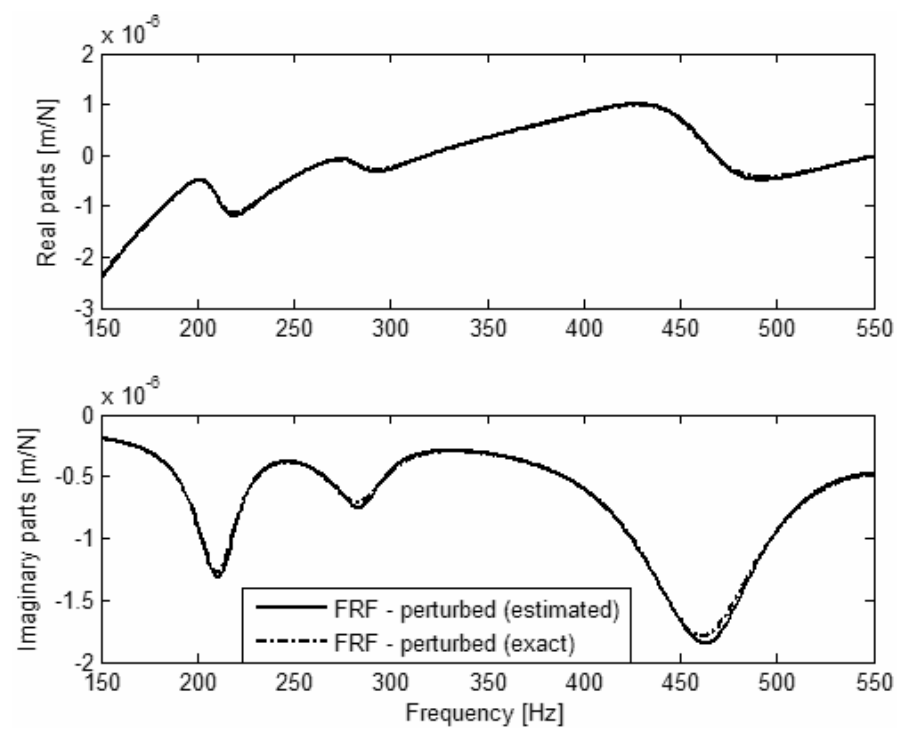

Fig. 10. Exact and first-order estimation of the FRF $H_{I I}(\omega, T, p)$ of the perturbed system by variations of the constraining layer thickness.

\section{Concluding remarks}

In this paper, the sensitivity analysis based on finite element models of damped structural systems containing viscoelastic materials has been addressed. A formulation has been developed for the computation of first-order derivatives of frequency response functions with respect to two different kinds of parameters, namely: the physical and/or geometrical structural parameters which appear explicitly in the finite element matrices, and temperature. Applications have been made to rectangular plates fully treated with constraining layers though the method can potentially be applied to other types of structural components.

As illustrated in the numerical applications presented, the sensitivities of complex frequency response functions convey valuable information about the influence of the design parameters on the dynamic behavior of the system, being also a very useful tool for the design, performance analysis and optimization of viscoelastic damping systems. 

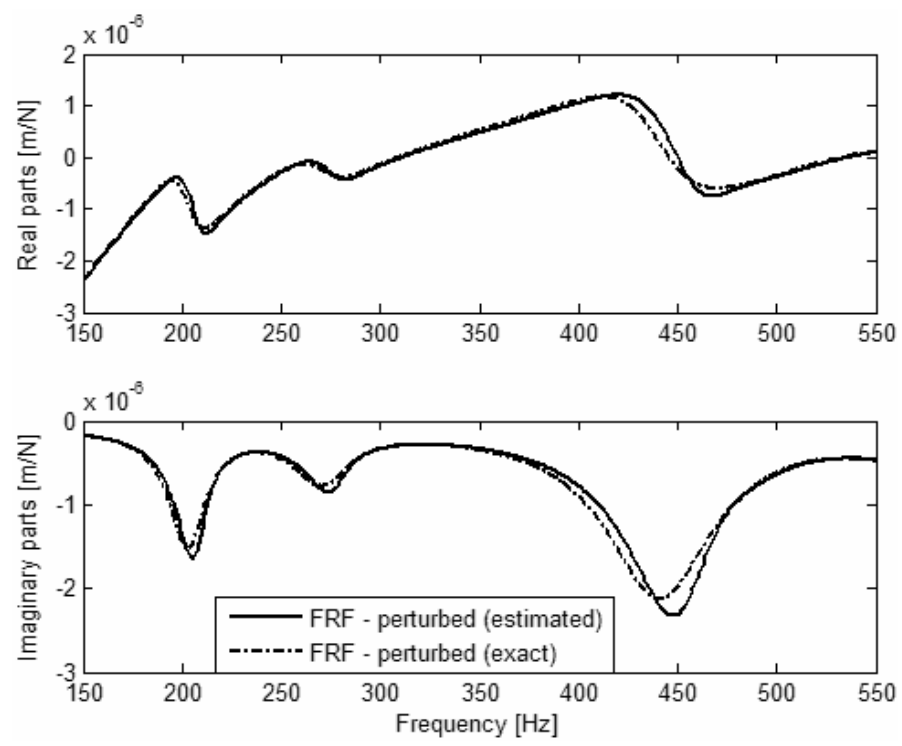

Fig. 11. Exact and first-order approximation of the FRF $H_{I I}(\omega, T, p)$ of the perturbed system by variation of temperature.

The use of the complex modulus approach, combined with the concepts of shift factor and reduced frequency justified by the principle of superposition frequency-temperature - has shown to be an adequate strategy to account for the typical dependency of the viscoelastic characteristics with respect to frequency and temperature in the finite element models of complex structural systems. However, the limitations of the first-order approximations in the prediction of response variations should be accounted for.

Another point that must be discussed is the use of the derivatives given by Eqs (31) and (32), in the computation of the sensitivities with respect to temperature. It should be remembered that those derivatives are obtained by deriving Eqs (28) and (29) which, in their turn, are obtained directly by curve-fitting of experimental data. As it is frequently observed when performing derivatives of experimental data, it is expected that the derivative functions Eqs (31) and (32) are less accurate than the fitted functions Eqs (28) and (29). Clearly, such errors can propagate to the computed values of the sensitivities with respect to temperature. This point requires further investigation.

\section{Acknowledgements}

A.M.G. Lima and M.H. Stoppa wish to thank Brazilian Agency CAPES for the grant of their graduate scholarships. D.A. Rade and V. Steffen Jr. are grateful to agency CNPq for the support to their research work.

\section{References}

[1] A.D. Nashif, D.I.G. Jones and J.P. Henderson, Vibration Damping, John Wiley \& Sons, New York, 1985.

[2] D.J. Mead, Passive Vibration Control, Wiley, Canada, 1998, 554.

[3] M.D. Rao, Recent Applications of Viscoelastic Damping for Noise Control in Automobiles and Commercial Airplanes, USA Symposium on Emerging Trends in Vibration and Noise Engineering, India, 2001.

[4] B. Samali and K.C.S. Kwok, Use of Viscoelastic Dampers in Reducing Wind- and Earthquake-induced Motion of Building Structures, Engineering Structures 17(9) (1995), 639-654.

[5] R.L. Bagley and P.J. Torvik, A Generalized Derivative Model for an Elastomer Damper, The Shock and Vibration Bulletin (49) (1979), 135-143.

[6] R.L. Bagley and P.J. Torvik, Fractional Calculus - A Different Approach to the Analysis of Viscoelastically Damped Structures, AIAA Journal 21(5) (1983), 741-748.

[7] R.L. Bagley and P.J. Torvik, Fractional Calculus in the Transient Analysis of Viscoelastically Damped Structures, AIAA Journal 23(6) (1985), 918-925. 
[8] D.F. Golla and P.C. Hughes, Dynamics of Viscoelastic Structures - A Time Domain, Finite Element Formulation, Journal of Applied Mechanics 52 (1985), 897-906.

[9] D. MacTavish and P.C. Hughes, Modeling of Linear Viscoelastic Space Structures, Journal of Vibration and Acoustics 115 (1993), $103-115$.

[10] G.A. Lesieutre, Finite Element for Dynamic Modeling of Uniaxial Rods with Frequency dependent Material Properties, International Journal of Solids and Structures 29(12) (1992), 1567-1579.

[11] G.A. Lesieutre and E. Bianchini, Time Domain Modeling of Linear Viscoelasticity Using Anelastic Displacement Fields, Journal of Vibration and Acoustics, Transations of the ASME 117 (1995), 424-430.

[12] G.A. Lesieutre and U. Lee, A Finite Element for Beams Having Segmented Active Constrained Layers With Frequency-dependent Viscoelastics, Smart Materials \& Structures 5(5) (1996), 615-627.

[13] E. Balmès, Model Reduction for Systems with Frequency Dependent Damping Properties, 15th International Modal Analysis Conference (IMAC) (1997).

[14] E. Balmès, Damping and Complex Modes, Modal Topics Presentation of the 21th International Modal Analysis Conference (IMAC), 2003.

[15] A.M.G. Lima and D.A. Rade, Modeling of Structures Supported on Viscoelastic Mounts Using FRF Substructuring, Proceedings of the Twelfth International Congress on Sound and Vibration, ICSV12, Lisbon, Portugal, 2005 (on CD-ROM).

[16] C.H. Park and A. Baz, Vibration Control of Bending Modes of Plates Using Active Constrained Layer Damping, Journal of Sound and Vibration 227(4) (1999), 711-734.

[17] M.A. Trindade and A. Benjeddou, Hybrid Active-Passive Damping Treatments Using Viscoelastic and Piezoelectric Materials: Review and Assessment, Journal of Vibration and Control 8(6) (2002), 699-746.

[18] J.J. de Espíndola, G.A.C. Mendéz, M.O. Lopes and C.A. Bavastri, Design of Optimum Viscoelastic Vibration Absorbers Based on Fractional Calculus Model, Proceedings of the XI International Symposium on Dynamic Problems of Mechanics, Ouro Preto, Brazil, 2005.

[19] R.M. Christensen, Theory of Viscoelasticity: An Introduction, Academic Press, Inc., New York, 2 nd edition, 1982.

[20] E.J. Haug, K.K. Choi and V. Komkov, Design Sensitivity Analysis of Structural Systems, Academic Press, 1986.

[21] A. Haldar and S. Mahadevan, Reliability Assessment Using Stochastic Finite Element Analysis, John Wiley \& Sons, 2000.

[22] J. Mothershead and M. Friswell, Finite Element Model Updating in Structural Dynamics, Kluwer Academic Publishers, Dordrecht, 1995.

[23] D.V. Murthy and R.T. Haftka, Derivatives of Eigenvalues and Eigenvectors of a General Complex Matrix, International Journal for Numerical Methods in Engineering 26 (1988), 293-311.

[24] C. Proppe, H.J. Pradlwarter and G.I. Schuëller, Equivalent Linearization and Monte Carlo Simulation in Stochastic Dynamics, Probabilistic Engineering Mechanics 18 (2003), 1-15.

[25] R.L. Fox and M.P. Kapoor, Rates of Change of Eigenvalues and Eigenvectors, AIAA Journal 6 (1968).

[26] R.B. Nelson, Simplified Calculation of Eigenvector Derivatives, AIAA J 14 (1976).

[27] B. Prasad and J.F. Emerson, A General Capability of Design Sensitivity for Finite Element Systems, AIAA/ASME/ASCE/AHA 23rd Structures, Structural Dynamics and Materials Conference. 1982.

[28] T.P. Khatua and Y.K. Cheung, Bending and Vibration of Multilayer Sandwich Beams and Plates, International Journal for Numerical Methods in Engineering 6 (1973), 11-24.

[29] M.H. Stoppa, Numerical Modeling and Optimal Design of Viscoelastic Damping Systems Applied to Rectangular Plates, Doctorate Thesis, Federal University of Uberlândia, School of Mechanical Engineering, 2003, Uberândia, Brazil (in Portuguese).

[30] M.L. Drake and J. Soovere, A Design Guide for Damping of Aerospace Structures, AFWAL Vibration Damping Workshop Proceedings 3 (1984). 

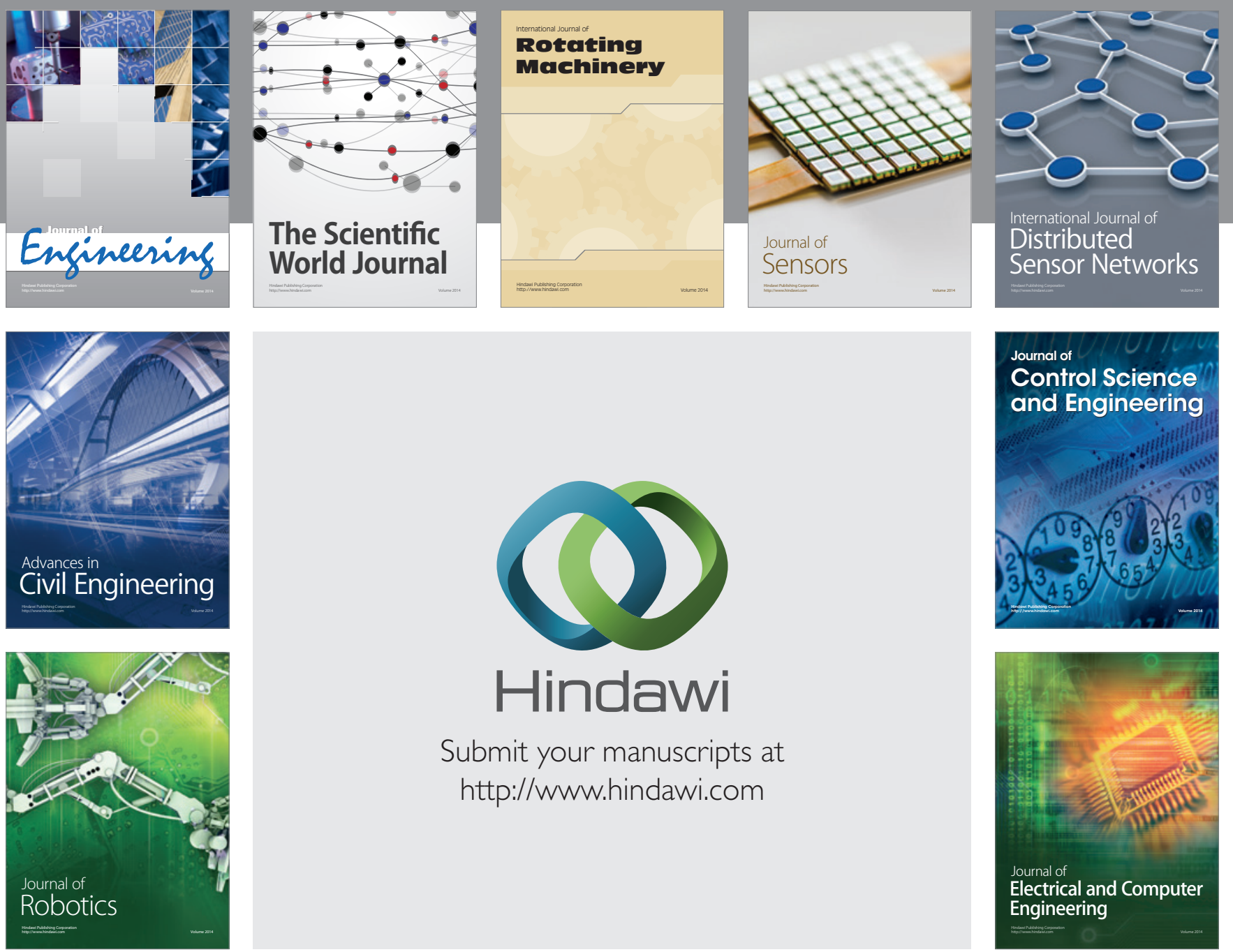

Submit your manuscripts at

http://www.hindawi.com
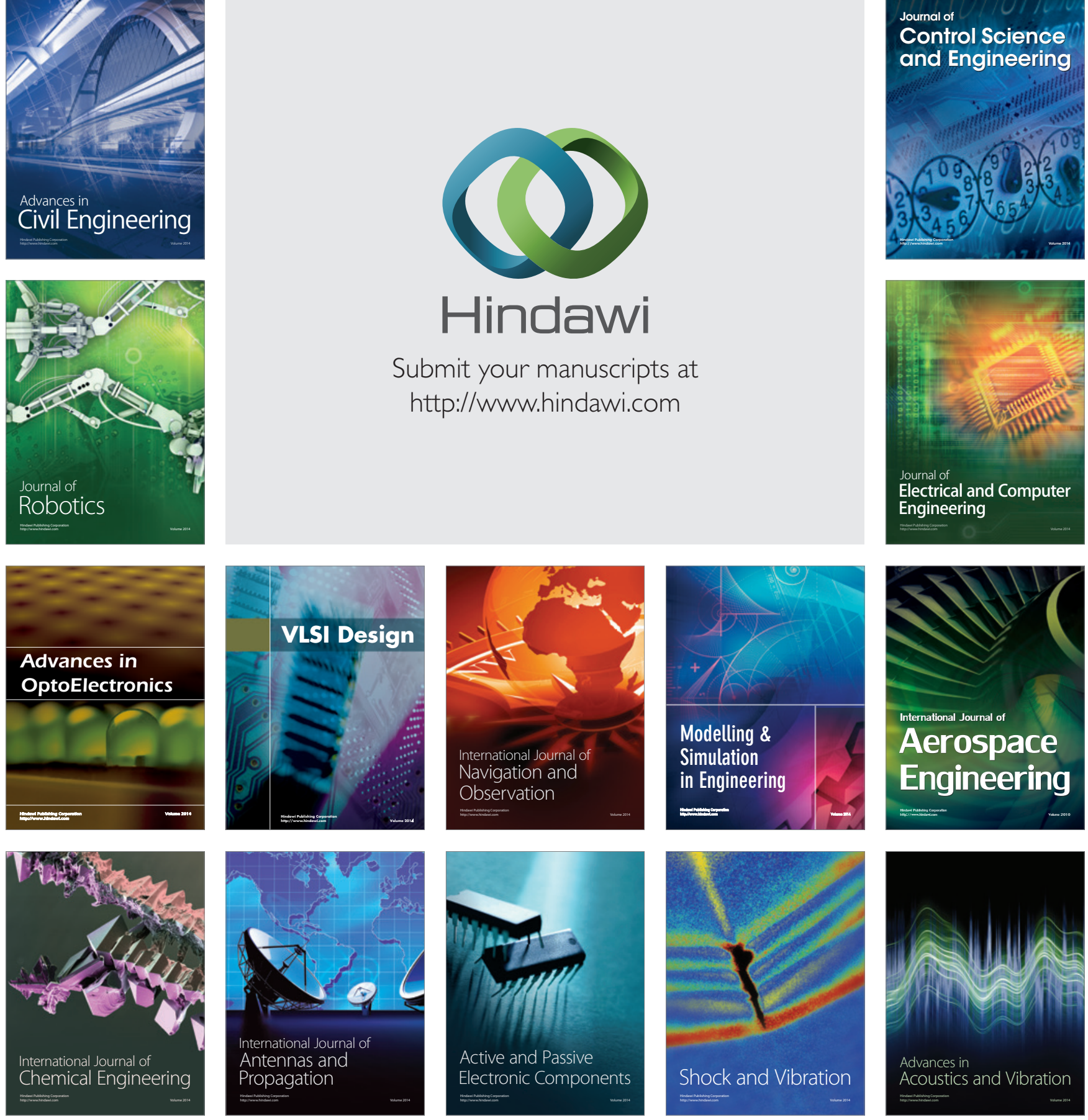